Meta

Journal des tradlucteurs

Translators' Journal

\title{
La place de la réflexion théorique dans l'apprentissage de la traduction
}

\section{Denis Juhel}

Volume 30, numéro 3, septembre 1985

URI : https://id.erudit.org/iderudit/003274ar

DOI : https://doi.org/10.7202/003274ar

Aller au sommaire du numéro

Éditeur(s)

Les Presses de l'Université de Montréal

ISSN

0026-0452 (imprimé)

1492-1421 (numérique)

Découvrir la revue

Citer cette note

Juhel, D. (1985). La place de la réflexion théorique dans l'apprentissage de la traduction. Meta, 30(3), 292-295. https://doi.org/10.7202/003274ar d'utilisation que vous pouvez consulter en ligne.

https://apropos.erudit.org/fr/usagers/politique-dutilisation/ 
BLOC-NOTES

\section{LA PLACE DE LA RÉFLEXION THÉORIQUE DANS L'APPRENTISSAGE DE LA TRADUCTION*}

Que ce soit dans les cours de version générale ou spécialisée, le professeur consacre une grande partie de son temps, la plus grande partie du temps parfois, à corriger les fautes de langue les plus diverses. Dans cet article cependant, je fais abstraction des fautes qui relèvent de la connaissance des langues de départ (LD) ou d'arrivée (LA), pour ne retenir que les seules faiblesses qui procèdent non de la compétence mais de la performance de l'étudiant, sachant, d'ailleurs, qu'il est souvent difficile de tracer une ligne de démarcation entre l'une et l'autre catégories.

De façon très empirique et sommaire, je définirai "fautes de performance" celles que l'étudiant de traduction (de son propre aveu très souvent) ne ferait pas s'il rédigeait spontanément dans sa langue maternelle (sans passer par la traduction donc). En d'autres termes, ce qui m'intéresse ici ce sont les points sur lesquels l'étudiant achoppe, points qui, en situation unilingue, font partie de son bagage langagier actif. Que l'attraction du texte de départ (TD) soit à l'origine de ces fautes, voilà un truisme de lon gue date. Alors pourquoi les ouvrages qui dressent la liste des interférences linguistiques les plus courantes ne permettent-ils que très partiellement au traducteur (apprenti ou de métier) de se détacher de TD ? Une première explication semble s'imposer : ces ouvrages correctifs se situant sur le plan de la compétence, ils sont de peu d'effet sur la qualité du texte d'arrivée (TA), plan de la performance.

Voyons d'abord quelques exemples des fautes en question. Parmi les plus typiques, relevées dans des cours de version économique ou de révision de traductions générales, je distinguerai trois grandes catégories à l'aide de quelques exemples : les impropriétés, les maladresses d'expression et l'appauvrissement lexical et stylistique de TA

1. Les impropriétés : ce sont principalement les métaphores incorrectes. Il suffit que l'image de TD se prolonge sur quelques lignes, ou que le verbe soit séparé d'un ou plusieurs compléments par une incidente plus ou moins longue, pour aboutir à coup sûr à l'impropriété. Par exemple, "Abattre / élimi- ner les barrières douanières " devient * diminuer les barrières" dans " les pays industrialisés devront diminuer leurs taxes à l'importation et *autres barrières ». (On aura deviné que dans TD le même verbe régissait les deux compléments.)

2. Les maladresses d'expression : il faudrait citer in extenso des phrases entières; ce serait un peu fastidieux et d'autant moins utile que " consommateurs " de traductions ou professeurs savent par expérience combien les lourdeurs, les charabias et les contorsions syntaxiques entravent souvent la lecture du TA.

3. L'appauvrissement lexical et stylistique du TA : il s'agit de mots et tournures que l'on rencontre rarement dans les traductions parce que la formulation de TD ne les suggérait pas. Je donnerai trois exemples. La charnière " or ", pourtant amplement utilisée tant en français écrit qu'oral, est absente des traductions des apprentis-traducteurs. Autre exemple, d'ordre lexical celui-ci, to slow down / reduce / decrease (en langue économique), qui ne génèrent que leur équivalent littéral ralentir/réduire/ diminuer, là où le dépouillement des textes français donne une grande variété de formulations (syntagme verbal ou nominal) : recul / coup d'arrêt ; marquer le pas / un ralentissement ; mettre un frein ; "décrocher $" .$. Un dernier exemple, de type syntaxique, l'interrogation rhétorique ( $M$. Untel ne confiait-il pas à un intime... " ou "Que penser de ces nouvelles mesures ? "), procédé ignoré des étudiants.

Les exemples des ressources inutilisées, ou insuffisamment exploitées, pourraient être multipliés ad nauseam. Carences, dira-t-on, qui relèvent de la stylistique comparée ou, pour sacrifier à la terminologie en vogue, de problèmes rédactionnels. Oui, si l'on se trouvait en situation unilingue, mais non, en fait, puisque ces carences, je l'ai précisé au tout début, apparaissent uniquement ou plus fréquemment en situation bilingue et ont donc un caractère spécifique et occasionnel. Force est de conclure que les observations grammaticales et stylistiques des professeurs et des manuels, sans être inutiles, ne sont pas de force à provoquer le déblocage qui permettrait au traducteur d'exploiter pleinement ses connaissances et les ressources stylistiques à sa disposition. Car il est clair qu'il ne suffit pas à l'étudiant de mettre le doigt sur les faiblesses évoquées plus haut pour s'en 
corriger par la suite ; d'autant que le professeur n'est pas souvent à même de faire la distinction entre les fautes de langue dues à l'ignorance, et les fautes de traduction proprement dites (dont je m'occupe ici) lorsque les premières sont formellement semblables aux secondes. Comment, donc, faire passer les connaissances langagières des (apprentis-) traducteurs du plan apparemment passif au plan actif? En d'autres termes, comment provoquer ou simuler la situation unilingue au cours de l'opération traduisante?

La réflexion théorique me semble un moyen privilégié pour opérer ce déblocage chez l'étudiant. Réflexion centrée sur l'appréhension du sens ou, ce qui revient au même, sur la distinction entre le respect de la forme et du sens et la conservation de la forme.

La justification d'une approche théorique dans le travaux pratiques de version est double. Elle mérite d'être présentée aux étudiants dès le premier cours, d'abord pour dissiper les craintes que le seul mot théorie soulève, ensuite parce que l'exposé de la méthode d'enseignement devrait faire partie d'un contrat pédagogique entre adultes. La suite de cet article s'articulera en trois mouvements : a) justification d'un enseignement pratique faisant largement appel à la théorie (c'est, en quelque sorte, la théorie de la théorie) ; b) forme sous laquelle peut se présenter la théorie du professeur, et comment elle doit aboutir chez l'étudiant à une théorisation; $c$ ) en dernier lieu, j'indiquerai quelques types d'exercices d'application qu'il est utile d'intégrer aux travaux pratiques de version.

Premièrement donc, quelques arguments pour défendre la légitimité de la théorie dans les cours de version. On sait la méfiance des étudiants et de certains professeurs pour ce qui ose s'affubler du titre " théorie ", sous le prétexte que la traduction fait partie d'un enseignement professionnel, donc pratique par définition. Si le terme fait peur, il est loisible à chacun de le remplacer par une définition : explication la plus complète et la plus cohérente possible de sa méthode, de ses prises de position ou de ses choix. Car je ne parle pas ici d'une théorie éthérée, coupée de la réalité. Bien au contraire, je ne veux que d'une théorie qui s'en nourrisse pour mieux éclairer la pratique. Parce que chaque fois qu'il prend la plume, le traducteur doit faire des choix, et ces choix successifs, si le traducteur est conséquent, ne procèdent-ils pas d'une théorie plus ou moins consciente ou articulée?

Enfin, dans le milieu universitaire où se situent nos écoles ou nos programmes de traduction, la théorie, dans son sens le plus général ou le plus restreint, n'a guère besoin de justifier sa place. Mais il s'agit moins de sacrifier à la tradition universitaire que d'utiliser de façon optimale un temps réel d'apprentissage très court ( 400 heures au plus de travaux pratiques, réparties sur 3 ans). Ne pas privilégier la réflexion serait quasiment réduire les cours de version à une suite de recettes empiriques. Le professeur n'a vraiment pas d'autre choix. Car si c'est en forgeant qu'on devient forgeron, l'adage n'avertit pas l'apprenti que, sans conscience, la pratique forcenée produit au moins autant de mauvais que de bons forgerons.

Dans un deuxième temps, voyons comment peut s'articuler une théorie de la traduction dans la classe de version. Si les exemples qui suivent expriment une perception particulière de l'opération traduisante, le lecteur pourra dégager les principes qui les soustendent et substituer ses exemples aux miens.

La pédagogie de la traduction ne devrait pas différer de l'enseignement d'autres matières au moins sur un point : le contrat pédagogique par lequel le professeur établit avec ses étudiants les objectifs du cours et les moyens pour les réaliser. Je passerai sur les règles matérielles, qu'il faut savoir adapter aux différents groupes et modifier au cours des années, pour ne parler que des objectifs qu'il faudrait très clairement énoncer au premier cours. Il serait injuste de demander à l'étudiant de découvrir par lui-même, et à ses risques et périls, les exigences d'un professeur avec lequel il ne passe, en règle générale, qu'un ou deux trimestres.

Reconnaissant que la vérité est multiple et que je ne peux présenter qu'une facette de cette vérité (tout au moins si ma théorie est solide), je m'efforce d'énoncer le plus tôt possible mes exigences. Je pose l'axiome premier : la traduction ne doit pas être un facteur d'anglicisation. De là suivront une série de mises en garde : je fais une chasse impitoyable aux anglicismes de tout acabit, je pénalise l'antéposition abusive de l'adjectif, les néologismes doivent être le no man's land du traducteur, etc. Ne pas hésiter non plus à faire connaître, bien avant la remise du premier devoir, ses préférences stylistiques, avec ce qu'elles ont de subjectif. Par exemple, face à lorsque le syntagme ne signifie rien d'autre que devant, et n'étant pas attesté par les "bons auteurs ", face à a le don de m'horripiler. Bien entendu, tant que les étudiants ne demandent pas des justifications qui dépassent l'exemple particulier, ces règles du jeu demeurent des "manies-de-prof; à-éviter-pour-obtenirune-bonne-note ". Idéalement, les demandes de justification, les dicussions devraient être spontanées, mais si les questions tardent, il faudra non seulement se justifier ponctuellement, mais regrouper ses points de vue dans une explication globale de l'opération traduisante (dimension sociolinguistique de la traduction par exemple, ou problèmes et rôle spécifiques du traducteur canadien, etc.). En l'absence de discussions, les étudiants se contenteraient de compiler une sorte de "Petit-Juhel", et l'on retomberait simplement dans le domaine des recettes empiriques que j'ai dénoncées plus haut.

Il est certaines mises en garde que je ne saurais donner sans les faire suivre immédiatement de quelques commentaires ou explications : ainsi, lorsque j'avertis mes étudiants qu'une traduction plus longue de $15 \%$ ou plus que l'original a 99 chances sur 100 d'être médiocre. (Selon quel principe ou loi linguistique ce qui se conçoit bien en anglais devraitil se dire longuement en français ?) Car ici, on touche au problème théorique fondamental que j'ai évoqué précédemment, c'est-à-dire l'appréhension du sens, 
et sa réexpression. Les théoriciens, linguistes et paralinguistes ont si communément répandu l'idée qu'il faut souvent étoffer en français pour rendre la tournure anglaise, que les traducteurs perdent de vue la concision propre au français ; les traducteurs qui oublient comme ces théoriciens que la traduction ne porte ni sur des syntagmes ni sur des phrases isolées, mais sur l'ensemble du message. Vos étoffements ont-ils souvent été contestés? Mais combien l'ellipse, l'implicite de la Langue et surtout de la Parole semble gêner les étudiants ! Ainsi, un texte sur la crise pétrolière utilisait huit fois en vingt-trois lignes le mot oil ; la version française que $j$ 'en proposais trois fois (une fois chaque pétrole, brut et pétrolier), deux fois si j'avais su résister aux objections des étudiants que ma concision mettait mal à l'aise, très peu d'entre eux ayant fait l'économie de plus d'un ou deux pétrole.

Le concept d'implicite linguistique ou sémantique n'est pas trop difficile à faire saisir lorsqu'on peut l'illustrer d'un mot ou d'un syntagme précis, comme dans l'ensemble précédent oil/pétrole. Lorsqu'il y a " lacune" dans l'une ou l'autre langue cependant, l'appréhension du sens est beaucoup plus ardue pour l'étudiant. Ainsi, après avoir conseillé à mes étudiants de Version économique de s'inspirer de tel ou tel document français avant de s'attaquer à leur traduction, une étudiante m'a avoué que ses lectures ne l'aidaient guère..., alors que le texte de référence traitait du même sujet. Et, en effet, tant que l'étudiant $n$ 'aura pas réfléchi sur la nature du sens, tant qu'il n'en aura pas saisi le caractère discontinu, il cherchera simplement les équivalences terme à terme et passera à côté des "autres façons de dire les choses" et du sens "entre les lignes". Dès lors, on comprend mieux pourquoi cette étudiante n'a pas su voir, entre autres, dans le seul terme "conjoncture " si fréquent en français économique (et politique) le sens que véhiculait toute une proposition du texte anglais; faut-il savoir lire de façon bien particulière pour voir dans les deux phrases suivantes le même propos : "Si sa clientèle diminue à cause de la conjoncture sectorielle, etc. " et "If the hatmaker's prospects have generally changed because of circumstances special to hats\%. (Cet exemple reproduit la traduction française que je proposais et la phrase anglaise correspondante.)

La théorie du professeur risquerait, je le répète, de s'ériger en principes rigides, ni plus ni moins utiles que les dictionnaires ou autres ouvrages normatifs, si le cours de version ne laissait pas suffisamment de place au dialogue. Sans vouloir enlever au professeur le rôle normatif qui est aussi le sien, on aura compris que la théorie dont je parle ici a avant tout valeur de maïeutique. Il faut, en effet, conduire l'étudiant à théoriser, c'est-à-dire organiser en un système cohérent dont il prend conscience les choix qu'il opère chaque fois qu'il traduit, ce qui présuppose de la part du professeur une présentation préalable du schéma global de la communication où se situe l'opération traduisante.
Dans l'optique de la théorie du sens appliquée à la traduction, soit encore "la théorie interprétative de la traduction ", pour citer D. Seleskovitch, deux exemples montreront aux étudiants que seule une perspective non linguistique offre une solution de traduction vraiment intelligible, et permet ensuite de réfuter le faux-problème d'une traduction qui serait idiomatique aux dépens de la fidélité au TD.

Exemple 1 : (Il s'agit de la traduction d'un script qui accompagne le défilé de diapositives, dans un cours audio-visuel.)

Ce que vous avez fait doit ressembler à cette image.

À l'impropriété près, la langue orale use souvent de tournures télescopées, la traduction est correcte, mais maladroite... comme toute autre solution qui ne s'inscrirait pas dans la dynamique du texte. Demander aux étudiants leurs solutions, en donnant toutes précisions utiles (l'apprenti-mécanicien, à ce point de la leçon, doit avoir monté sur la tronçonneuse une pièce nommée connecteur) et comparer leurs solutions avec la version révisée :

Cette diapositive vous montre le connecteur en place.

Exemple 2, emprunté à M. Pergnier, les Fondements sociolinguistiques de la traduction. (Pancarte lue à l'aéroport de Londres.)

Les passagers ne doivent pas s'avancer audelà de ce point de contrôle sans avoir vérifié au préalable près de la compagnie avec laquelle ils voyagent.

Transcodage peu intelligible pour l'unilingue ( $a$ fortiori pour le passager anxieux ou en retard), mais où le bilingue retrouvera la formulation de l'anglais. La formulation authentiquement française, elle, véhicule avec moitié moins de mots toute l'information de la première " traduction " et combien clairement!

Accès réservé aux passagers ayant effectué les formalités d'embarcation.

J'ai insisté sur la nécessité d'encourager les discussions de groupe; mais ce n'est pas là le seul moyen de théoriser. Je terminerai cet exposé en suggérant quatre types d'exercices systématiques que j'énoncerai dans leurs grandes lignes.

1. Exercices très variés visant à faire toucher du doigt la différence entre le mot et le sens, traduire et transcoder : voir le livre de Jean Delisle, l'Analyse du discours comme méthode de traduction.

2. Autre exercice pour éliminer le plus possible l'attraction de la formulation originale et faire découvrir empiriquement la nature du sens : la traduction à vue. Texte de 200 mots au plus, sans difficultés 
terminologiques. Les étudiants le lisent une fois, puis retournent la feuille. Un étudiant lit une fois, deux tout au plus, une longue phrase, ou deux plus courtes (total de trois lignes minimum). TD reste toujours caché pour le reste de la classe. Un deuxième étudiant est chargé de dire en français ce qu'il a retenu des lignes qui viennent d'être lues (selon le niveau des étudiants, cette phase serait précédée d'une " explication de texte " rapide servant à dégager les idées principales et les caractéristiques stylistiques de TA). Puis, avec la classe, l'étudiant reconstruit petit à petit (à l'aide d'un jeu de questions du professeur) la totalité de l'information véhiculée par l'énoncé anglais. Les solutions correctes, même incomplètes ou assez maladroites, sont inscrites au tableau, comparées et améliorées. Et ainsi de suite, la version française étant reprise, à la fin de l'exercice oral/écrit, pour en vérifier la cohésion, la logique interne. Ce n'est qu'en tout dernier lieu que l'on revient à TD pour s'assurer que rien n'a été perdu en cours de route. Dernière phase exceptée, la technique rapidement esquissée ici rappellera celle qu'on utilise en classe de langue seconde, voire de langue maternelle, pour la reconstitution orale d'un texte écrit, ou vice versa, pour les contractions de texte ou la technique du résumé.

3. Exercice pour démontrer qu'il n'existe pas une seule traduction correcte d'un texte, et que le style, le ton de l'ensemble sont en grande partie déterminés par la phrase d'introduction (cohésion du texte, logique interne). Faire traduire un paragraphe par la classe, à partir de deux ou trois solutions correctes (et différentes) pour la phrase d'introduction. Comparer et apprécier les deux ou trois versions parallèles.

4. Pour amener l'étudiant à rationaliser les solutions qu'il propose : justification et défense d'une traduction par une petite équipe ( 3 ou 4 ), avec contradiction par une autre équipe ou par l'ensemble de la classe.

Non seulement ces exercices, et d'autres que je laisse à l'imagination du lecteur, combinés avec la réflexion devraient-ils, comme tout enseignement digne de ce nom, apprendre à l'étudiant à apprendre, ils devraient également délier la langue souvent pâteuse de l'apprenti. Et décider une fois pour toutes le futur traducteur à ne jamais sacrifier le génie de sa langue de travail et l'intelligibilité de ses versions à la prétendue, ou mal comprise, fidélité au texte de départ.

\section{DENIS JUHEL}

- Reprend, en le développant, le thème d'un exposé présenté à uI atelier du colloque de l'ACLA à Chicoutimi (1982)

\section{OUVRAGES CITÉS}

DELISLE, Jean (1980) : l'Analyse du discours comme méthode de traduction, Université d'Ottawa.
JUHEL, Denis (1982) : Bilinguisme et traduction au Canada; rôle sociolinguistique du traducteur, Québec, CIRB, Université Laval.

PERGNIER, Maurice (1980) : les Fondements socilinguistiques de la traduction, Paris, Honoré Champion.

SELESKOVITCH, Danica (1976) : "Traduire : de l'expérience aux concepts", in Études de linguistique appliquée, vol. 24, Paris, Didier. 